PACS 87.50.U-, 87.64.km, 87.80.Dj, 87.85.Ox

\title{
Infrared and terahertz in biomedicine
}

\author{
F.F. Sizov \\ V. Lashkaryov Institute of Semiconductor Physics, NAS of Ukraine, \\ 41, prospect Nauky, 03680 Kyiv, Ukraine, e-mail:sizov@isp.kiev.ua
}

\begin{abstract}
A number of potential advances of infrared and terahertz technologies in application mainly to biomedicine is shortly discussed. In spite of the fact that there are a number well established imaging and spectroscopic techniques in application to biomedicine there exists some problems where IR and $\mathrm{THz}$ technologies are the challenging technologies that can provide information not available from other techniques. E.g., they can be applied in the cases where there is a need of improving the surgical removal of cancer, strictly locating tumor margins when conservation of normal tissue is needed.
\end{abstract}

Keywords: infrared and terahertz technologies, biomedical application.

Manuscript received 03.07.17; revised version received 14.08.17; accepted for publication 06.09.17; published online 09.10.17.

\section{Introduction}

Infrared (IR) and especially terahertz (THz) technologies have become one of the major fields of applied researches to a great degree driven by potential applications in biomedicine.

The IR technologies in applications to biomedicine are known since 1950s. Concerning the $\mathrm{THz}$ area, a simple search with keywords involving "terahertz" and the traditional terms "far infrared" and "sub-millimeter waves" comes up with thousands of hits (see, e.g., Fig. 1). Here, the current manuscript will be to a great degree limited, since the author considered, the most important developments in IR and $\mathrm{THz}$ technologies in application to biomedicine. Numerous groups have now demonstrated biomedical applications in distinguishing healthy tissue from tissue with a disease or injury resulting in modifications in protein structure or salt/protein content, in cells of breast cancer, colon cancer, burn imaging, and some other applications [1].

It can be noted that in 1990s the interest in $\mathrm{THz}$ biological and medical effects in the former Soviet Union Countries exceeded (at least by the number of publications) than that one in other countries [2]. Especially in the $\mathrm{THz}$ area, recently, there has been flurry of research of terahertz applications, which includes $\mathrm{THz}$ imaging and spectroscopy for biomedical, military, security, food and drug control applications, etc.

For a long time, the terahertz range was considered as a final frontier of the electromagnetic spectrum. It was mainly related with difficulties in generating terahertz radiation. Now, radiation frequencies between 0.2 and $10 \mathrm{THz}$ have become to a great extent the domain of optoelectronic laser-based techniques. Still other types of THz sources (IMPATT diodes, BWO sources, Gunn oscillators, quantum cascade lasers, gas and free-electron lasers, etc.) play an important role in $\mathrm{THz}$ technologies.

Basic progress in $\mathrm{THz}$ imaging and spectroscopy is related just with optoelectronic approaches that use either femtosecond lasers or diode lasers to get tunable $\mathrm{THz}$ radiation. Photomixers, photoconductive switches or nonlinear crystals convert the near-infrared laser radiation into terahertz waves, either broadband or spectrally resolved.

Scientific and particularly application activity in IR and $\mathrm{THz}$ technologies have increased significantly in recent two decades, and it is to be expected that the trends especially in THz science and technology will be only continued and extended. 


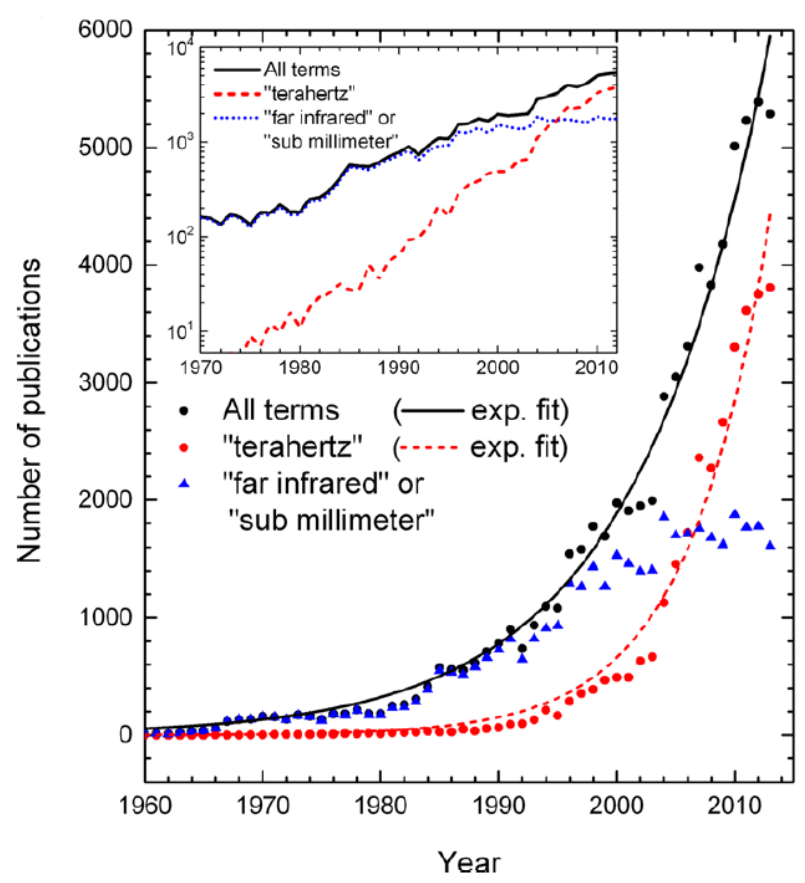

Fig. 1. Chronological chart (1960-2013) and exponential fits (1960-2012) for the numbers of publications with topics involving "terahertz" and the traditional terms "far infrared" and "sub-millimeter waves". The box shows the logarithmic plot between 1970 and 2012 [3].

In addition to the well-known technical opportunities, historical examination of Internet usage as well as the number of publications and patent applications, confirms ongoing interest in this technique (Fig. 1). Activity of $\mathrm{THz}$ technologies is noticed by annual growth rate in between $9 \%$ and $21 \%$ [3].

\section{Transparency and spectral regions}

A shift from the scientific to more application-oriented researches can be observed both for IR, THz and microwave spectral regions. As concerning the microwave region, the advantages of microwave remote sensing consist in all-weather, day-and-night imaging capacity as compared to IR remote sensing for which, e.g., cloudiness prevents the Earth surface observation, though the spatial resolution for this spectral region is much higher, and IR thermal remote sensing is the passive one as compared to microwave remote sensing that is active for Earth surface observation.

For $\mathrm{THz}$ region, atmospheric absorption is too large for remote sensing (see Figs. 2 and 3). Active microwave sensing (realized using radars) is based on the transmission of longwave microwaves $(\lambda \sim 3-25 \mathrm{~cm}, v \sim$ $10-1.2 \mathrm{GHz})$ through the atmosphere and then recording the amount of energy backscattered from the terrain.

Attention to energy consumption problems is raising the interest for the applications of thermal remote sensing in urban environments. E.g., advances in thermal infrared detectors and techniques have facilitated obtaining high-resolution thermal images over large areas, for a correct quantitative evaluation of land surface temperatures [5]. In the $\mathrm{THz}$ spectral region, the atmosphere absorption is high (Figs. 2 and 3), which prevents to apply long-distance $\mathrm{THz}$ technologies (see Fig. 4).

There is no commonly agreed definition of the upper and lower frequency limits of terahertz $\mathrm{THz}$ and infrared IR radiation and one can meet several different segmentations of IR and $\mathrm{THz}$ spectral regions. In many cases (see, e.g., [8]) today, the IR region is taken as the region with the wavelengths $\lambda$ approximately between 0.7 and $20 \mu \mathrm{m}$. From $\lambda \sim 0.7$ to $1.1 \mu \mathrm{m}$, it is the NIR (near

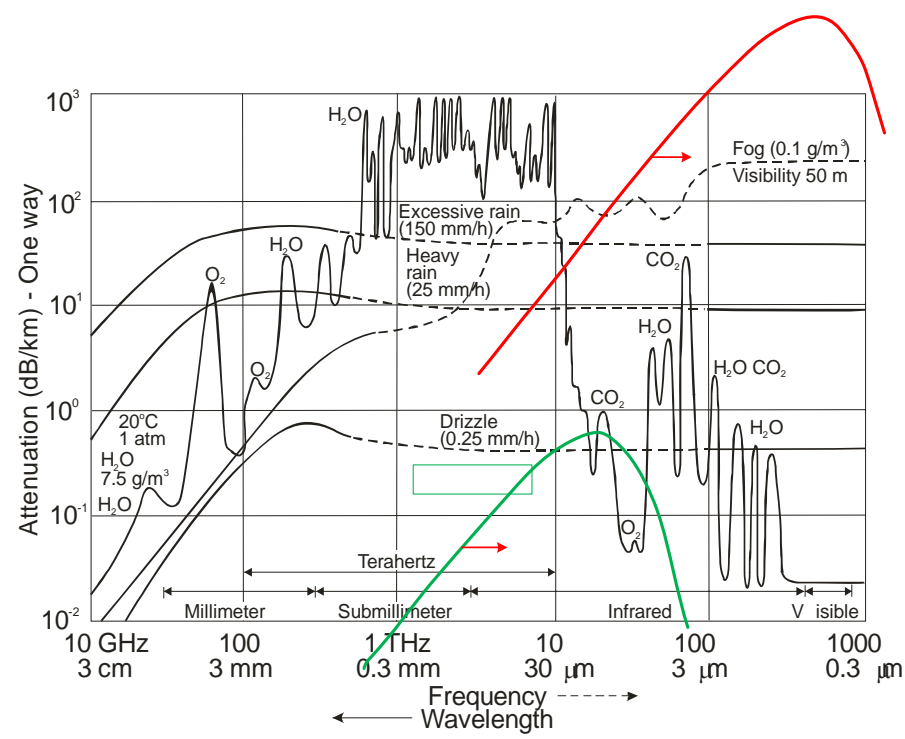

Fig. 2. Transparency of Earth atmosphere from visible to radiofrequency band region [4]. Also are shown spectral radiances for ideal black body for temperatures $T \approx 6000 \mathrm{~K}$ (Sun) and $T \approx 300 \mathrm{~K}$ (Earth surface). It is seen that strong water and oxygen absorption in the atmosphere make high altitude platforms essential for good seeing of space objects. 
IR) region, from $\lambda \sim 1.1$ to $2.5 \mu \mathrm{m}$ it is the SWIR (short wavelength IR) region, from $\lambda \sim 2.5$ to $7.0 \mu \mathrm{m}$ it is the MWIR (medium wavelength IR) region, from $\lambda \sim 7.0$ to $15.0 \mu \mathrm{m}$ it is the LWIR (long wavelength IR) region and from $\lambda>15.0 \mu \mathrm{m}$ is the VLWIR (very long wavelength IR) region. In other classifications, the IR spectra are taken for the wavelengths from $\lambda \sim 0.7$ to $1000 \mu \mathrm{m}$ $[9,10]$. Here, it is accepted that IR spectra lie within the wavelengths range $\lambda \sim 0.7$ to $30 \mu \mathrm{m}$. In most applicable for thermovision IR spectral regions 3 to $5 \mu \mathrm{m}$ and 7 to $14 \mu \mathrm{m}$, atmosphere is transparent (Fig. 5).

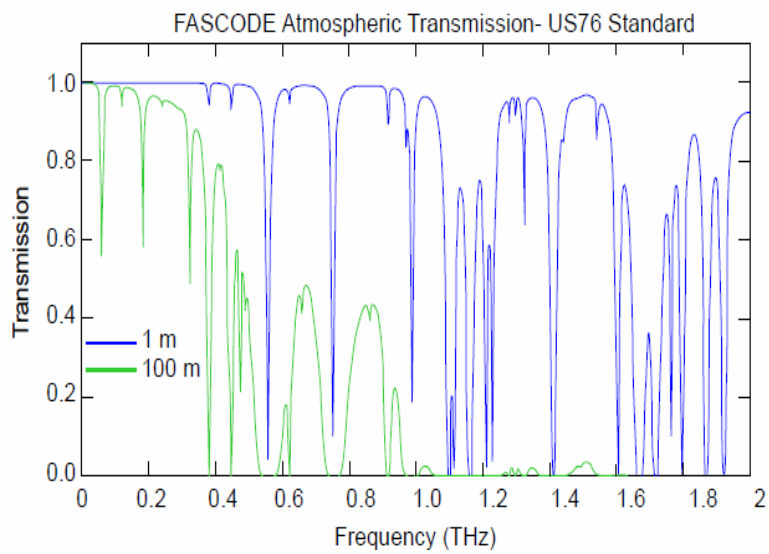

Fig. 3. Transmission of $\mathrm{THz}$ radiation in air [6]. In $\mathrm{THz}$ active imaging systems emission radiation frequencies should be inside an atmospheric transmission windows to avoid strong water vapor absorption.



Fig. 4. Predicted range to identify a concealed weapon using a single scanning spot sensor [7].

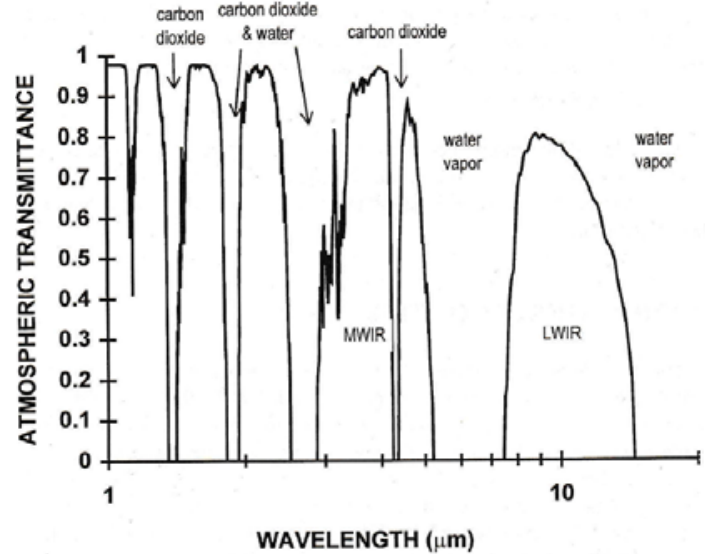

Fig. 5. Typical atmosphere transmission over $1 \mathrm{~km}$ path length [11].
As concerning the $\mathrm{THz}$ spectrum, there exist several classifications especially in the low frequency part of $\mathrm{THz}$ spectra - the millimeter-wave region. The millimeter-wave region of the electromagnetic spectrum is generally taken to range from $30 \mathrm{GHz}(\lambda=1 \mathrm{~cm})$ to $300 \mathrm{GHz}(\lambda=1 \mathrm{~mm})$ [12] and even wider [13], while the terahertz region is now frequently considered to be between the radiation frequencies $v=0.1 \mathrm{THz}$ $(\lambda=3 \mathrm{~mm})$ and $v=10 \mathrm{THz}(\lambda=30 \mu \mathrm{m})$ (see, e.g., [1417]), leaving the region of overlap between $0.1 \mathrm{THz}$ $(\lambda=3 \mathrm{~mm})$ and $0.3 \mathrm{THz}(\lambda=1 \mathrm{~mm})$.

Now frequently terahertz region is considered to be between $0.1 \mathrm{THz}$ and $10 \mathrm{THz}[1,17,18]$. Partial overlapping with $\mathrm{THz}$ range is frequently used as the term "far-infrared" [19, 20] (FIR, VLWIR), which is often taken from 100 to $1000 \mu \mathrm{m}$ (see, e.g., $[9,10]$ ). The range placed between frequencies from $300 \mathrm{GHz}$ to $3 \mathrm{THz}$ is usually called as the submillimeter-wave band [21], though in this paper the terms "terahertz" and "submillimeter-wave" are considered to be synonyms. Here, it is accepted that $\mathrm{THz}$ spectra lie within the radiation frequencies between 0.1 and $10 \mathrm{THz}$ (from $(\lambda=3 \mathrm{~mm}$ to $30 \mu \mathrm{m})$.

As to $\mathrm{THz}$ radiation frequency range, it is known that many materials that are opaque to visible and infrared light are transparent to $\mathrm{THz}$ radiation [22-24] (paper, foams, plastic, textiles, etc.). Attenuation values (in $\mathrm{dB}$ ) for some clothing, fabric, and building materials in the radiation range $94-1042 \mathrm{GHz}$ are presented in [25]. $\mathrm{THz}$ imaging was applied for non-destructive quality check of hidden damages in foams after accident in 2003 with Space Shuttle Columbia.

$\mathrm{THz}$ radiation is heavily absorbed by water. Another feature of $\mathrm{THz}$ radiation frequency is that the fundamental frequencies - rotational and vibrational modes of molecules like proteins or DNA - are located in this region (see, e.g., [21, 26, 27]). This gives the opportunity to control content of materials under investigation by using the known fingerprints of substances.

Because of the relatively large width of the $\mathrm{THz}$ spectra, which exceeds, for example, the visible light range $(\lambda \sim 0.4-0.7 \mu \mathrm{m})$ and is close to the wide band of IR radiation $(\sim 0.7-30 \mu \mathrm{m}), \mathrm{THz}$ radiation is of great importance in terms of fundamental researches as well as in technology and life sciences, as in this region rotational and vibrational lines of a lot of substances are located. All the applications require different $\mathrm{THz}$ detector sensitivities and source capabilities, closely related to temperature exploitation and different spectral band applicability. These circumstances should be taken into account in estimations of $\mathrm{THz}$ systems cost that may differ several orders.

\section{Applications}

Typical IR technologies applications can be separated in two major groups. On the one hand, there are NIR and

(C) 2017, V. Lashkaryov Institute of Semiconductor Physics, National Academy of Sciences of Ukraine 
SWIR spectral regions, which are commonly employed for the assessment of artworks (e.g., paintings and frescoes), since some painting pigments are semitransparent to the infrared in these spectral bands and some other are not (e.g., carbon based). In this region, also IR spectroscopy is applied in biomedical science. On the other hand, there is infrared thermography, which involves detection of the surface and subsurface layers of objects on the differences in thermal signatures in the MWIR and LWIR spectral bands. In biomedicine applications mostly thermography and spectroscopy are applied in any IR band [28].

$\mathrm{THz}$ waves are sufficiently short to provide resolution of less than $1 \mathrm{~mm}$ (far field approximation) and several hundred nanometers for solid-state objects [29] (near field approximation), yet long enough to penetrate most nonmetallic substances, such as materials used to make clothing, rucksacks and tarps [25]. As such, they are useful to security agents and military personnel alike for revealing concealed weapons, chemical explosives and biological agents. Besides, security applications, namely: airport screeners, higherresolution terahertz sensors, could provide enhanced identification of battlefield targets, better missile guidance and other combat advantages [3]. Soldiers, marines and fighter pilots are increasingly trained to use not only the visible wavelengths that their eyes can process, but infrared wavelengths as well, the application fields of which are widespread in military and civil domains. But contrary to IR region where imaging as a rule is passive, due to the lack of appreciable terahertz power in the thermal background, it is necessary to use an illumination ( $\mathrm{THz}$ sources) for imaging.

The increasing demand of unoccupied and unregulated bandwidth for wireless communication systems will lead to extension of operation frequencies toward the lower $\mathrm{THz}$ frequency range. Higher carrier frequencies will allow for fast transmission of huge amounts of data as needed for new emerging applications. Despite the tremendous hurdles that have to be overcome with regard to sources and detectors, circuit and antenna technology and system architecture to realize ultrafast data transmission in a scenario with extensive transmission loss, a new area of research beginto form [30-32].

Among the countries engaged in developing $\mathrm{THz}$ technologies for diverse applications today, USA, UK, Germany, China, South Korea, Japan, Russia, Taiwan and some others are leading. Among the countries developing their own cooled and uncooled IR FPA technologies for commercial and military applications, there are the USA, UK, Japan, Germany, South Korea, Canada, China, Italy, Russia, and in small quantities some others. IR and $\mathrm{THz}$ technologies today and in the nearest times will be among key technologies for security systems, communications, space, biomedical and food control applications, etc.
For insight in IR and $\mathrm{THz}$ applications, there exist a large number of books where these questions are developed deeply and thoroughly (see, e.g., [28, 3345]).

As a rule, biomedical imaging techniques are considered as non-invasive methods for looking inside the body without opening up the body surgically. There are many medical imaging techniques (see, e.g., Table 1), every technique has different risks, limitations and benefits and cannot be used as be individually reliable in all biomedical applications $[28,46]$.

First of all, development of IR and $\mathrm{THz}$ technologies are important in early cancer diagnostics, as a cancer is one of the leading causes of death worldwide. In 2012 there was 8.2 million death data vs. different form of cancer [48]. Cancer is the second leading cause of death and in 2015 was responsible for 8.8 million deaths - nearly 1 in 6 global death [49]. The total number of deaths due to cardiovascular disease read 17.3 million a year according to the WHO causes of death 2008 summary tables [50]. Thus, death data vs. different form of cancer are comparable to cardiovascular diseases and will continue to rise to over 13.1 million in 2030 [51] and the economic impact of cancer is significant and increasing. The total annual economic cost of cancer in 2010 was estimated at approximately US\$ 1.16 trillion [49].

Among women, the breast cancer decease is one of the prime causes of their death worldwide $[42,50]$.

Breast cancer patients diagnosed are characterized into three cases: in the first case $90 \%$ patients diagnosed will undergo surgery to treat the disease, in the second case, $60 \%$ will undergo breast conserving surgery, according to breast conserving surgery the primary tumor is removed with a margin of normal tissue around it and remaining mastectomy. Around $10-15 \%$ of patients will require the second operation, as the margins are not free of cancer on histopathology [52]. That's why more accurate technique is needed to assess resection margins during surgery to avoid the next operation. In the case of $\mathrm{THz}$ technology for cancer diagnostics, it is conditioned with strong water absorption as water concentration reveal a lot about the health of human tissue, with water content in cancerous cells higher than in healthy cells.

Thermography has a potential in screening the breast cancer diagnostics detecting the growth of malignant tumor due to increase of the internal temperature captured by thermograms. Infrared thermography has emerged in recent years as an attractive and reliable technique to address complex nondestructive (NDT) problems [53]. But in its applications one should take into account that medical infrared imaging can only be applied by physicians who have been educated and trained intensively as well as have received a medical certificate [54]. This is still valid now. 
Table 1. A comparison between the different biomedical imaging techniques (following [46]).

\begin{tabular}{|c|c|c|c|c|c|c|}
\hline \multirow[b]{2}{*}{$\begin{array}{l}\text { Imaging } \\
\text { technique }\end{array}$} & \multicolumn{2}{|c|}{ Image quality } & \multicolumn{2}{|c|}{ System availability } & \multicolumn{2}{|c|}{ Safety } \\
\hline & $\begin{array}{l}\text { Spatial } \\
\text { resolution }\end{array}$ & $\begin{array}{l}\text { Good } \\
\text { contrast }\end{array}$ & Cost & $\begin{array}{l}\text { Real time } \\
\text { information }\end{array}$ & $\begin{array}{l}\text { Ionizing } \\
\text { radiation } \\
\text { effect }\end{array}$ & $\begin{array}{l}\text { Heating } \\
\text { effect }\end{array}$ \\
\hline $\begin{array}{l}\text { Radiography } \\
\text { (X-ray) }\end{array}$ & $1 \mathrm{~mm}$ & $\begin{array}{l}\text { Soft tissue } \\
\text { and fluid }\end{array}$ & Medium & No & Yes (active) & Low \\
\hline CT (X-ray) & $0.5 \mathrm{~mm}$ & $\begin{array}{l}\text { Hard and } \\
\text { soft tissues }\end{array}$ & High & No & Yes (active) & Low \\
\hline $\begin{array}{l}\text { MRI (magnetic } \\
\text { and radio } \\
\text { frequency fields) }\end{array}$ & $0.5 \mathrm{~mm}$ & $\begin{array}{l}\text { Hard and } \\
\text { soft tissues }\end{array}$ & High & No & No (active) & Medium \\
\hline Ultrasonography & $1 \mathrm{~mm}$ & Soft tissues & Low & Yes & No (active) & Negligible \\
\hline Elastography ${ }^{1)}$ & $200 \mu \mathrm{m}$ & Soft tissues & Medium & Yes & No (active) & Low \\
\hline Optical & $100 \mathrm{~nm}$ & Soft tissues & Low & No & No (active) & Medium \\
\hline Radionuclide & $3 \mathrm{~mm}$ & Soft tissues & High & No & Yes (active) & Medium \\
\hline $\begin{array}{l}\text { IR } \\
\text { (Thermography) }\end{array}$ & $1.5 \mathrm{~mm}^{2)}$ & Soft tissues & Low & No & $\begin{array}{l}\text { No } \\
\text { (passive) }\end{array}$ & No (passive) \\
\hline $\mathrm{THz}$ & $\begin{array}{l}\sim 50-1000 \mu \mathrm{m} \\
\text { (dependent on } \\
\text { wavelength) }\end{array}$ & Soft tissues & $\begin{array}{l}\text { High/Medium } \\
\left(\mathrm{TDS} / \mathrm{CW}^{3)}\right)\end{array}$ & No & No (active) & $\begin{array}{l}\text { Dependent } \\
\text { on radiation } \\
\text { power }\end{array}$ \\
\hline
\end{tabular}

1) Elastography imaging may be ultrasound elasticity imaging, magnetic resonance elasticity imaging, optical elasticity imaging or tactile imaging.

2) Far-field approximation.

${ }^{3)} \mathrm{CW}$ systems with photomixers.

The US Federal Communications Commission established maximum permissible exposure limits of $1 \mathrm{~mW} / \mathrm{cm}^{2}$ for 6 $\mathrm{min}$ in the $30-300 \mathrm{GHz}$ frequency range [47].

$\mathrm{CT}$ is the computerized tomography, MRI is the magnetic resonance Imaging, IR is the infra-red, $\mathrm{THz}$ is the terahertz, TDS is the time delay spectroscopy, CW is the continuous wave. Active means the presence of radiation source, passive means the analysis of radiation that is emitting from the object examined (e.g., thermography).

Today, potential and existing IR and $\mathrm{THz}$ technology applications are broad in such diverse fields as medicine, telecommunications, energy control, space research, missile systems, and defense IR signature analysis and measurement techniques, territory control and security, etc. IR and $\mathrm{THz}$ technologies are well developed for astronomy, military and surveillance applications, Earth surface control, heat stress diagnostics in industrial processes, thermal conductivity of samples with IR thermography, breast cancer diagnostics, thermography in semiconductor reliability testing, control in building enclosures and art, tank levels of liquids, automotive applications, gas analyzers (see, e.g., [55-59]) and in biomedical applications mainly for breast cancer diagnostics both in preclinical research settings as well as in the clinical assessment of patients (see, e.g., [28, 44, 45, 53, 60]).

In biomedical applications, IR technologies may serve as one of the additional imaging methods (limited as a primary breast cancer diagnostic $[42,46,61])$, as compared to other better developed techniques. Moreover, interpretations of thermographic images depend on the specialists, which may lead to errors and uneven results [42].

Application of $\mathrm{THz}$ waves to biomedical questions is motivated by: (1) strong absorbance by water; (2) rotational and vibration resonances in biomolecules; and (3) low possibility of objects damage by low-energy photons that unlike X-rays are not so harmful to the tissues (see, e.g., [21, 45, 52]). However, many of the reported $\mathrm{THz}$ effects under low-intensity radiation produce a variety of bio-effects [2].

$\mathrm{THz}$ spectroscopy and imaging have a drawback because of limited spatial resolution. Diffraction limit (far field approximation) prevents light of a wavelength $\lambda$ from being focused to a spot (Airy disk diameter) with a diameter of $\varnothing \approx 2.44 \lambda(f / D)$ where $f / D=\mathrm{F} \#$ is the $f$ number of the optical system, $f$ is the focus length and $D$ is the diameter of lens. At radiation frequency of $1 \mathrm{THz}$ and $\mathrm{F} \#=1$ the Airy disk diameter $\varnothing \approx 0.7 \mathrm{~mm}$, 
therefore, THz spectroscopy and $\lambda(f / D)$ and microscopy of objects is restricted to measurements of ensembles of molecules. Imaging resolution will be restricted by dimensions $(l \approx 1.22 \lambda(f / D))$ over sample heterogeneity. Therefore, there were developed some of microscopy techniques that extend the scope of $\mathrm{THz}$ spectroscopy, among which, e.g., there is scattering-type near-field $\mathrm{THz}$ microscopy (see, e.g., [29, 62]).

In spite of great efforts in recent two or three decades resulting in an exponential growth of the number of publications $[1,3,6,36,38,39] \mathrm{THz}$ applications in general are still in early stage of development, so many other potential applications in future can be added to astronomy, biomedicine, pharmaceutical, wireless communications, security, package inspection, food industry, material studies, spectroscopy, etc. (see Fig. 6). Also, THz absorption on diluted soil samples can be effective in identifying soil constituents, such as aromatic compounds, and soil contaminants, such as pesticides [63].

\section{Influence of radiation}

THz waves have low photon energy to ionize atoms and molecules, and this energy is much less to cause cancer and genetic mutations. Other health effects can be caused by thermal effects (temperature changes during irradiation) under powerful $\mathrm{THz}$ radiation. But as it was summarized, a couple decades ago that many of reported $\mathrm{THz}$ effects under low-intensity radiation produce a variety of bio-effects, a lot of which are quite unexpected and can't be explained by temperature changes during irradiation [2].
As concerning the nervous tissue, only few studies were performed in application to a living organism for evaluationof transient effects in these spectral regions. Using $60 \mathrm{GHz}$ low-density short time $(5 \mathrm{~s})$ radiation power $\left(\sim 4 \mathrm{~mW} / \mathrm{cm}^{2}\right)$ incident on the object, it was shown that the real-time effects in isolated leech ganglions could not mimicked by equivalent bath heating [65]. Therefore, it seems that the needs of $\mathrm{THz}$ radiation effects researches on living organisms for determination of $\mathrm{THz}$ and $\mathrm{mm}$ waves ultimate dosage and exposure time are important for evaluation of the transient effects on the health and for elaboration of safety standards.

$\mathrm{THz}$ imaging for human breast cancer diagnostics is now less advanced as compared to thermography, and further development of the technology and clinical examination are needed to evaluate its feasibility in the clinical environment [66]. Also, when carrying out $\mathrm{THz}$ breast cancer diagnostics, it should be taken into account the power level of radiation that can be harmful for living objects as $\mathrm{THz}$ diagnostics is mainly possible for active vision systems.

Widely-spread TDS spectroscopy and imaging pulsed $\mathrm{THz}$ systems for biomedical applications has started just after the publication [67] due to the sensitivity of $\mathrm{THz}$ radiation absorption (see Figs. 1 and 2 ) to water content in tissue. The degree of hydration of tissue could be used as a measure of disease state [67]. The typical spectral range of pulsed $\mathrm{THz}$ systems, as a rule, is within $v \sim 0.1-4.0 \mathrm{THz}$ with the peak frequency $\sim 0.4 \mathrm{THz}$ [12]. For pulsed systems the typical $\mathrm{THz}$ source radiation power is lower or close to $0.1 \ldots 1 \mu \mathrm{W}$ in dependence of illuminated power, radiation frequency, semiconductor material used as a switch, etc.

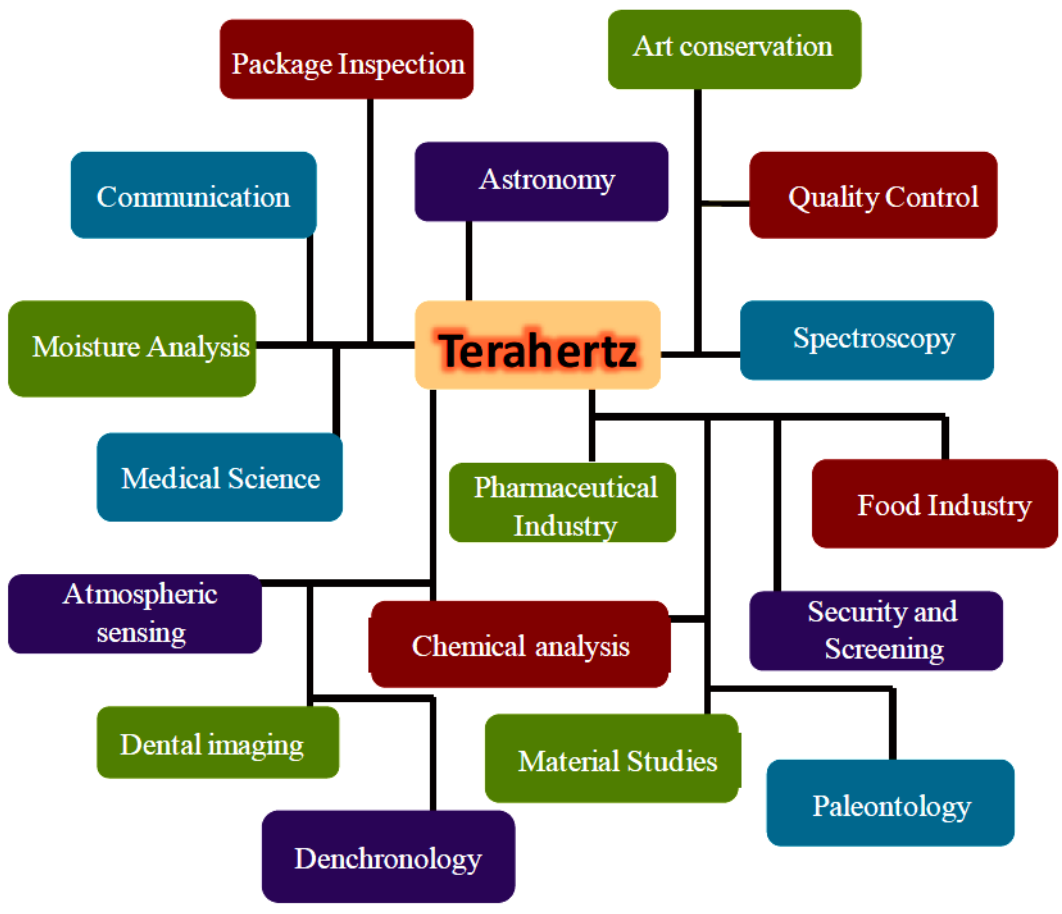

Fig. 6. General scheme of explored and future $\mathrm{THz}$ spectroscopy and imaging applications [64]. 
Beside pulsed systems, there exist several possible schematics of $\mathrm{THz}$ continuous wave (CW) instrumentation. In the typical $\mathrm{CW}$ system a single fixed radiation frequency or tuned in narrow frequency band sources (e.g., IMPATT diodes, Gunn oscillators, quantum cascade lasers (QCLs), BWO sources, gas lasers, etc.) can be used. Broadband ( $v \sim 0.1 \ldots 2.5 \mathrm{THz})$ radiation is possible to obtain applied photomixers, where two lasers emit in two adjacent spectral ranges in visible or near-IR regions. The photomixer translates the laser beams of two lasers into $\mathrm{THz}$ radiation (frequency domain $\mathrm{THz}$ radiation). For this kind of $\mathrm{CW}$ systems, the radiation $\mathrm{THz}$ power, as a rule, doesn't exceed $2 \mu \mathrm{W}$ in dependence of radiation frequency, semiconductor material used as a photomixer, etc.

$\mathrm{CW}$ imaging allows for a more compact and simple systems, while pulsed imaging measurements yield a broader range of information, as it is also possible to register the phase data in them.

In spite of potential capabilities at the moment, $\mathrm{THz}$ imaging is limited to a great degree, as it could distinguish the tumors and normal tissues, serious inflamed and normal livers, but could not distinguish the grade of tumors and inflammation [68].

Although $\mathrm{THz}$ waves do not penetrate far into the body (from several hundred micrometers up to several millimeters in dependence of fatty content), that is one of the main drawbacks, it would be possible to project $\mathrm{THz}$ endoscopically, as in optical coherence tomography. Examples of diagnosis of cancerous tissues with $\mathrm{THz}$ radiation are reported as they exhibit different hydration levels from normal tissues [69, 70].

Terahertz spectra of tissues with a high water content, e.g. skin, follow a similar trend as that for pure water but the absorption is not as high [1]. Water absorption limits the depth of tissue that may be imaged using terahertz to a few hundred micrometers in skin and up to several millimetersis tissue with more fat content like breast.

Because of strong absorption by water in many cases of applications to biomedical and food, $\mathrm{THz}$ imaging and spectroscopic systems can be applied, e.g., for mapping tumor margins or surface of food products with not a great depth but about $2 \mathrm{~mm}$ in breast because fatty tissue [71, 72]). Moreover it was found the frequency dependence of absorption coefficient on salt, protein and DNA content. In addition $\mathrm{THz}$ water absorption depends on protein structural changes, such as ligand binding or denaturing [1].

Among the $\mathrm{THz}$ imaging and spectroscopy systems for these purposes, there are mainly used such systems as the $\mathrm{THz}$ pulse imaging - $\mathrm{THz}$ time domain spectroscopy (TDS), and continuous wave (CW) photomixer systems (see, e.g., [51]).

Today $\mathrm{THz}$ spectroscopy and imaging used in the radiation frequency range $\sim 0.1-4 \mathrm{THz}$ require femtosecond lasers and photoconductive broadband antenna sources and detectors both for generation and detection of $\mathrm{THz}$ radiation, respectively. They are the most expensive components of the systems, and this is a reason of healthcare cost as compared to other methods. Still, the challenges of $\mathrm{THz}$ spectroscopy and imaging applications lie in providing information not available from other methods.

It was shown that an advance for enhancing $\mathrm{THz}$ biomedical imaging can be reached by introducing exogenous contrast agentsthat are capable to improve the contrast. It was found that the $\mathrm{THz}$ reflection can be increased in the cancer cells with gold nanoparticles [45] upon their irradiation with an IR laser, or magnetic induction heating of superparamagnetic iron oxide nanoparticles [73], due to a rise in the temperature of local water.

Biomedical diagnosis is based on both structural and on functional data, as well as patient history and subjective symptoms. Structural imaging methods are as following: X-ray, ultrasound imaging, MRI (magnetic resonance imaging), and the classical and electron microscopy also fall into the imaging category.

Medical diagnostic with IR waves is a functional, passive and non-invasive method for analyzing physiological functions related to body thermal homeostasis or organ temperature and, therefore, its results cannot be directly compared to the results obtained by structural imaging methods. It is used to detect and locate temperature distribution characterized by a non-physiological increase or decrease in temperature at the body surface. Today, the improved hardware detection systems with additional advanced software solutions make it possible to incorporate atomical and physiological information by image fusion, which helps to generate information of affected areas. IR thermography is used for measuring and analyzing physiological functions and pathology related to the body thermal homeostasis and temperature [74].

Since the middle of 20-th century, infrared (IR) spectroscopy coupled to microscopy (IR microspectroscopy) has been recognized as a nondestructive, label free, highly sensitive and specific analytical method with many potential useful applications in various fields of biomedical researches and, in particular, cancer research and diagnosis. Although many technological improvements have been made to facilitate biomedical applications of this powerful analytical technique, it has not yet properly come into the scientific background of many potential end users. Therefore, to achieve these fundamental objectives an interdisciplinary approach is needed with basic scientists, spectroscopists, biologists and clinicians who must effectively communicate and understand each other's requirements and challenges [59].

A lot of daily-used materials, such as clothing, dry paper, plastics, dry leather etc., are transparent in this wavelength range $[22,23]$ (paper, foams, plastic, textiles, etc.). Many plastics are used now for 3D printed $\mathrm{THz}$ optics [24] especially in CW systems. Table 2 presents the data for some plastic and other materials in the THz spectral region. 
Table 2. Parameters of some materials in the $\mathrm{THz}$ region $(T=300 \mathrm{~K})$ important for $\mathrm{THz}$ optics.

\begin{tabular}{|l|l|l|l|}
\hline Material & Refractive index, $\boldsymbol{n}$ & Absorption coefficient, $\mathbf{c m}^{\mathbf{- 1}}$ & Reference \\
\hline ABS & 1.57 & $\begin{array}{l}\approx 1.5-25 \\
0.2-1.5 \mathrm{THz}\end{array}$ & {$[24]$} \\
\hline PLA & 1.89 & $\begin{array}{l}\approx 1.5-25 \\
0.2-0.9 \mathrm{THz}\end{array}$ & {$[24]$} \\
\hline Nylon & 1.72 & $\begin{array}{l}\approx 0.2-40 \\
0.2-1.2 \mathrm{THz}\end{array}$ & {$[24]$} \\
\hline Bendlay & 1.53 & $\begin{array}{l}\approx 1.5-25 \\
0.5-1.5 \mathrm{THz}\end{array}$ & {$[24]$} \\
\hline Polystyrene & 1.56 & $\approx 0-4.5$ & $0.2-1.5 \mathrm{THz}$ \\
\hline HDPE & 1.53 & $\begin{array}{l}\approx 0 \\
0.2-1.5 \mathrm{THz}\end{array}$ & {$[24]$} \\
\hline PP & $\begin{array}{l}\approx 0.1 \\
0.2-1.5 \mathrm{THz}\end{array}$ & {$[24]$} \\
\hline High-resistance Si & 3.42 & $<0.05$ \\
& & $0.1-2.0 \mathrm{THz}$ & {$[24]$} \\
\hline Quartz & 1.96 & $\begin{array}{l}\approx 1-9 \\
0.1-2.0 \mathrm{THz}\end{array}$ & {$[77]$} \\
\hline TPX & $\approx 1.45,0.3-2.5 \mathrm{THz}$ & $0.2-0.9,0.3-2.5 \mathrm{THz}$ & {$[75]$} \\
\hline Teflon-AF & $\approx 1.39,0.3-2.5 \mathrm{THz}$ & $\approx 0.2-3.4,0.3-2.5 \mathrm{THz}$ & {$[75]$} \\
\hline HDPE & $\approx 1.53,0.3-2.5 \mathrm{THz}$ & $\approx 0-2.4,0.3-2.5 \mathrm{THz}$ & {$[75]$} \\
\hline Teflon & $\approx 1.435,0.3-2.5 \mathrm{THz}$ & $\approx 0-2.4,0.3-2.5 \mathrm{THz}$ & {$[75]$} \\
\hline
\end{tabular}

Here, ABS is the acrylonitrile butadiene styrene, PLA - polyactic acid, HDPE - high-density polyethylene, PP - polypropylene, TPX - methyl-pentene copolymer (polymethylpentene), HDPE - high density polyethylene.

For some explosives, the data about absorption coefficients are presented in [78].

The fact that $\mathrm{THz}$ radiation has a shorter wavelength than microwave radiation and thus has the capability of having a higher spatial resolution, and at low levels of radiation power does not pose any known harm to living organisms, makes $\mathrm{THz}$ imaging a powerful and presumably safe imaging technology [12, $45,65]$.

Unlike the pulsed $\mathrm{THz}$ imaging, the $\mathrm{CW}$ imaging presented only yields intensity data and does not provide any depth, frequency-domain or time-domain information about the subject when a fixed-frequency source and a single detector or arrays of detectors are used. However, the CW imaging systems are sufficient for many imaging applications affording a compact, simple, fast and relatively low-cost system. Since it does not require a pump-probe system and the complexity of the optics involved, the optics of CW systems are much simpler and thus their cost can be considerably reduced as compared to pulsed $\mathrm{THz}$ systems [46, 79], and since it does not require a time delay scan, image formation can take place more quickly [12].

Compared to IR and microwave systems, $\mathrm{THz}$ imaging, spectroscopy and communication systems and their components (detectors, sources, etc.) are still remaining less developed. That is a reason why at the moment $\mathrm{THz}$ wave instrument capabilities are still away in comparison, e.g., with IR or microwave system feasibilities.

For insight in applications, there exist a large number of books in which the topics pointed out shortly are developed deeply and thoroughly (see, e.g., $[9,28$, 33-39].

\section{Conclusion}

IR and $\mathrm{THz}$ technology applications today are broad in such domains as astronomy, military and surveillance, telecommunications, security, energy and food control, but it seems one of the most topical are biomedical applications, e.g., for breast cancer diagnostics, colon cancer, burn imaging, etc., especially in the cases where there is a need of accurate location of tumor margins when conservation of normal tissue is required. It can be expected that potentiality of these technologies will be only in progress in diverse directions in biological and medical fields. Further work is needed for scientific challenge to provide information not available from other techniques. One of the main barriers in providing healthcare conclusions by using $\mathrm{THz}$ technologies is the cost of $\mathrm{THz}$ pulse imaging and spectroscopy instrumentation that is mostly related with a high cost of short pulse lasers needed for their applications. 


\section{References}

1. Dhillon S.S., Vitiello M.S., Linfield E.H. et al. (32 Co-Authors). The 2017 terahertz science and technology roadmap. J. Phys. D: Appl. Phys. 2017. 50. P. 043001.

2. Pakhomov A.G., Akyel Y., Pakhomova O.N., Stuck B.E., and Murphy M.R. Current state and implications of research on biological effects of millimeter waves: A review of literature. Bio Electro Magnetics. 1998. 19, No. 7. P. 393-413.

3. Hochrein T. Markets, availability, notice, and technical performance of terahertz systems: Historic development, present, and trends. J. Infrared, Millimeter, Terahertz Waves. 2015. 36. P. 235-254.

4. Lettington A.H., Blankson I.M., Attia M., and Dunn D. Review of imaging architecture, Proc. SPIE. 2002. 4719. P. 327-340.

5. Bitelli G., Conte P., Csoknyai T., Franci F., Girelli V.A. and Mandanici E. Aerial thermography for energetic modeling of cities. Remote Sens. 2015. 7, No. 2. P. 2152-2170.

6. Rogalski A., and Sizov F. Terahertz detectors and focal plane arrays. Opto-Electr. Rev. 2011. 19, No. 3. P. 346-404.

7. Assessment of millimeter-wave and terahertz technology for detection and identification of concealed explosives and weapons. Committee on Assessment of Security Technologies for Transportation (2007), www.nap.edu.

8. Holst G. Common Sense Approach to Thermal Imaging. SPIE Optical Eng. Press, Bellingham, 2000.

9. Maldague X. Theory and Practice of Infrared Technology for Nondestructive Testing. N.Y., US, Wiley, 2001.

10. Ibarra-Castanedo C., Bendara A., Maldague X.P.V. Infrared vision applications for the nondestructive testing of materials. 5-th Pan American Conf. for NDT, October 2-6, 2011, Cancun, Mexico.

11. Holst G.C. Electro-optical Imaging System Performance. SPIE Optical Eng. Press, Bellingham WA, USA. 2003.

12. Karpowicz N., Zhong H., Xu J., Lin Kuang-I., Hwang J.-S., and Zhang X.-C. Comparison between pulsed terahertz time-domain imaging and continuous wave terahertz imaging. Semicond. Sci. Technol. 2005. 20. P. S293-S299.

13. Chattopadhyay G. Submillimeter-wave coherent and incoherent sensors for space applications, in: Sensors, eds. S.C. Mukhopadhyay and R.Y.M. Huang. Springer, New York, 2008. P. 387-414.

14. Crowe T.W., Bishop W.L., Porterfield D.W., Hesler J.L., and Weikle R.M. Opening the terahertz window with integrated diode circuits. IEEE J. Solid-State Circuits. 2005. 40. P. 21042110 .
15. Chan W.L., Deibel J. and Mittleman D.M. Imaging with terahertz radiation. Rep. Prog. Phys. 2007. 70. P. 1325-1379.

16. Dragoman D. and Dragoman M. Terahertz fields and applications. Prog. Quant. Electron. 2004. 28. P. 1-66.

17. Hintzsche H. and Stopper H. Effects of terahertz radiation on biological systems. Critical Rev. Environmental Sci. Technol. 2012. 42. P. 2408-2434.

18. Sizov F., Rogalski A. THz detectors. Progr. Quant. Electr. 2010. 34. P. 278-347.

19. Blaney T.G. Signal-to-noise ratio and other characteristics of heterodyne radiation receivers. Space Sc. Rev. 1975. 17. P. 691-702.

20. Richards P. Bolometers for infrared and millimeter waves. J. Appl. Phys. 1994. 76. P. 1-24.

21. Siegel P.H. Terahertz technology in biology and medicine. IEEE Trans. Microwave Theory Techn. 2004. 52, No. 10. P. 2438-2447.

22. Zhao G., Mors M., Wenckebach T. and Planken P. Terahertz dielectric properties of polystyrene foam. J. Opt. Soc. Am. B. 2002. 19, No. 6. P. 1476-1479.

23. Hartwick T.S., Hodges D.T., Barker D.H., and Foote F.B. Far infrared imagery. Appl. Opt. 1976. 15. P. 1919-1922.

24. Busch S., Weidenbach M., Fey M., Schafer F., Probst T., and Koch M. Optical properties of 3D printable plastics in the THz regime and their application for 3D printed THz optics. J. Infrared, Millimeter, Terahertz Waves. 2014. 35. P. 993-997.

25. Gatesman A.J., Danylov A., Goyette T.M. et al. Terahertz behavior of optical components and common materials. Proc. SPIE. 2006. 6212. P. 6212OE.

26. Loffler T., Siebert K., Czasch S., Bauer T., and Roskos H.G. Visualization and classification in biomedical terahertz pulsed imaging. Phys. Med. Biol. 2002. 47. P. 3847-3852.

27. Nagel M., Forst M., and Kurz H. THz biosensing devices: fundamentals and technology. J. Phys. Condens. Matter. 2006. 18. P. S601-S618.

28. Bronzino J.D., and Peterson D.R. Biomedical Engineering Handbook - Biomedical Signals, Imaging, and Informatics (4-th Ed.). CRC Press, Boca Raton, 2017.

29. Chen H.-T., Kersting R., and Cho G.Ch. Terahertz imaging with nanometer resolution, Appl. Phys. Lett. 2003. 83, No. 15. P. 3009-3011.

30. Federici J. and Moeller L. Review of terahertz and subterahertz wireless communications. 2010. J. Appl. Phys. 107. P. 111101.

31. Kleine-Ostmann T. and Nagatsuma T. A review on terahertz communications research. J. Infrared, Millimeter, Terahertz Waves. 2011. 32. P. 143171.

32. Akyildiz I., Jornet J., Han C. Terahertz band: Next frontier for wireless communications. Phys. Commun. 2014. 12. P. 16-32. 
33. Seeing Photons: Progress and Limits of Visible and Infrared Sensor Arrays. Committee on Developments in Detector Technologies; National Research Council, ISBN 978-0-309-15304-1. 2010.

34. The RF and Microwave Handbook, Editor in Chief Mike Golio. CRC Press LLC, USA, Boca Raton, 2001.

35. Sensing with Terahertz Radiation, Ed. D. Mittleman. Berlin-Heidelberg-New York, Springer Verlag, 2003.

36. Bründermann E., Hübers H.-W., and Kimmitt M.F. Terahertz Techniques. Springer, Heidelberg, 2011.

37. Yun-Shik Lee, Principles of Terahertz Science and Technology. Springer Science+Business Media, LLC, 2009.

38. Handbook of Terahertz Technology for Imaging, Sensing and Communications, Ed. D. Saeedkia. Oxford, Cambridge, Philadelphia, New Delhi, Woodhead Publishing Limited, 2013.

39. Terahertz Spectroscopy and Imaging, Eds. KaiErik Peiponen, J. Axel Zeitler, Makoto KuwataGonokami. Springer-Verlag, Berlin-Heidelberg, 2013.

40. Haynie D.T. Biological Thermodynamics. Cambridge University Press, Cambridge, 2001.

41. Ring E.F.J., Ammer K. The technique of infrared imaging in medicine. Thermol. Int. 2000. 10. P. 7 14.

42. Raghavendra U., Acharya U.R., Ng E.Y.K., Tan J.H., and Gudigar A. An integrated index for breast cancer identification using histogram of oriented gradient and kernel locality preserving projection features extracted from thermograms. Quantitative InfraRed Thermography J. 2016. 13, No. 2. P. 195 209.

43. Corsi C. New frontiers for infrared. Opto-Electron. Rev. 2015. 23, No. 1. P. 3-25.

44. Medical Infrared Imaging: Principles and Practices, Eds. M. Diakides, J.D. Bronzino, and D.R. Peterson. CRC Press, Boca Raton, 2013.

45. Terahertz Biomedical Science and Technology, Ed. J.-H. Son. CRC Press, Boca Raton, 2013.

46. Kasban H., El-Bendary M.A.M., and Salama D.H. A comparative study of medical imaging techniques. Int. J. Information Sci. Intelligent System. 2015. 4, No. 2. P. 37-58.

47. Chou C.K., Andrea J. (Eds.), IEEE Standard for Safety Levels with Respect to Human Exposure to Radio Frequency Electromagnetic Fields, $3 \mathrm{kHz}$ to $300 \mathrm{GHz}, \mathrm{C} 95.1-2005,2006$.

48. http://www.cancerresearchuk.org/healthprofessional/cancer-statistics/worldwide-cancer.

49. World Health Organization Fact Sheet, 2017, http://www.who.int/features/factfiles/cancer/en/.

50. http://www.world-heartfederation.org/cardiovascular-health/global-factsmap/.

51. Panwar A.K., Singh A., Kumar A., and Kim H. Terahertz imaging system for biomedical applications: Current status. Int. J. Eng. Technol. 2013. 13. P. 33-39.

52. Pickwell E. and Wallace V.P. Biomedical applications of terahertz technology. J. Phys. D: Appl. Phys. 2006. 39. P. R301-R310.

53. Khodayar F., Sojasi S., and Maldague X. Infrared thermography and NDT: 2050 horizon. Quantitative InfraRed Thermagraphy. 2016. 13, No. 2. P. 210-231.

54. Berz R., and Sauer H. The medical use of infraredthermography. History and recent applications. Thermografie-Kolloquium-2007, Vortrag 04, 1-12, 2007

(www.ndt.net/search/docs.php3?MainSource=61).

55. Jha A.R. Infrared Technology: Applications to Electro-Optics, Photonic Devices and Sensors. Wiley, N.Y., 2000.

56. Rieke G.H. Infrared detector arrays for astronomy. Annu. Rev. Astro. Astrophys. 2007. 45. P. 77-115.

57. Ratches J.A. Current and future trends in military night vision applications. Ferroelectrics. 2006. 342. P. 183-192.

58. Cozzolino D. and Murray I. A review on the application of infrared technologies to determine and monitor composition and other quality characteristics in raw fish, fish products, and seafood. J. Appl. Spectroscopy Rev. 2012. 47, No. 3. P. 207-218.

59. Bellisola G., Sorio C. Infrared spectroscopy and microscopy in cancer research and diagnosis. Am. J. Cancer Res. 2012. 2, No. 1. P. 1-21.

60. Jiang L.J., Ng E.Y.K., Yep A.C.B., Wu S., Pan F., Yau W.Y., Chen J.H., and Yang Y. A perspective on medical infrared imaging. J. Med. Eng. \&Techn. 2005. 29, No. 6. P. 257-267.

61. Head J.F. and Elliott R.L. Infrared imaging: making progress in fulfilling its medical promise. IEEE Eng. Medicine \& Biology Mag. 2002. 21. P. 80-85.

62. Eisele M., Cocker T.L., Huber M.A., Plankl M., Viti L., Ercolani D., Sorba L., Vitiello M.S., and Huber R. Ultrafast multi-terahertz nanospectroscopy with sub-cycle temporal resolution. Nature Photonics. 2014. 8. P. 841-845.

63. Ayesha Y. Imagerie Térahertz $2 D$ et $3 D$ : Application pour l'étude des matériaux du patrimoine culturel. These, L'Universite de Bordeaux, 2011.

64. Lewis R.A. Terahertz transmission, scattering, reflection, and absorption: The interaction of $\mathrm{THz}$ radiation with soils. J. Infrared, Millimeter, Terahertz Waves. 2017. 38, No. 7. P. 799-807.

65. Romanenko S., Siegel P.H., Wagenaar D.A., and Pikov V. Effects of millimeter wave irradiation and equivalent thermal heating on the activity of individual neurons in the leech ganglion. $J$. Neurophysiol. 2014. 112, No. 10. P. 2423-2431.

66. Peter B.St., Yngvesson S., Siqueira P., Kelly P., Khan A., Glick S., and Karellas A. Development

(C) 2017, V. Lashkaryov Institute of Semiconductor Physics, National Academy of Sciences of Ukraine 
and testing of a single frequency terahertz imaging system for breast cancer detection. IEEE J. Biomed. Health Inform. 2013. 17, No. 4. P. 785-797.

67. $\mathrm{Hu}$ B.B. and Nuss M.C. Imaging with terahertz waves. Opt. Lett. 1995. 20, No. 16. P. 1716-1718.

68. Zhang C.H., Zhao G.F., Jin B.B., Hou Y.Y., Jia H.H., Chen J., and Wu P.H. Terahertz imaging on subcutaneous tissues and liver inflamed by liver cancer cells. Terahertz Sci. Technol. 2012. 5, No. 3. P. 114-123.

69. Nakajima S., Hoshina H., Yamashita M., Otani C. and Miyoshi N. Terahertz imaging diagnostics of cancer tissues with a chemometrics technique. Appl. Phys. Lett. 2007. 90. P. 041102.

70. Brun M.A., Formanek F., Yasuda A., Sekine M., Ando N. and Eishii Y. Terahertz imaging applied to cancer diagnosis. Phys. Med. Biol. 2010. 55. P. 4615-4623.

71. Fitzgerald A.J., Wallace V.P., Jimenez-Linan M., Bobrow L., Pye R.J., Purushotham A.D. and Arnone D.D. Terahertz pulsed imaging of human breast tumors. Radiology. 2006. 239. P. 533-540.

72. Grachev Y.V., Kuklin I.A., Gerasimov I.V., Smolyanskay O.A., Kozlov S.A., and Bespalov V.G. Study of how radiation of the frequency range $0.05-2 \mathrm{THz}$ affects biological tissues of various thickness in medical diagnosis. J. Opt. Technol. 2010. 77, No. 11. P. 731-733.

73. Zhang R., Zhang L., Wu T., Zuo Sh., Wang R., Zhang C., Zhang J., and Fang J. Contrast-enhanced continuous-terahertz-wave imaging based on superparamagnetic iron oxide nanoparticles for biomedical applications. Opt. Express. 2016. 24, No. 8. P. 257234.

74. Szentkuti A., Kavanagh H.S., Grazio S. Infrared thermography and image analysis for biomedical use. Periodicum Biologorum. 2011. 113, No. 4. P. 385-392.

75. Kawamura J., Paine S., and Papa D.C. Spectroscopic measurements of optical elements for submillimeter receivers. Proc. 7-th Intern. Symp. on Space Terahertz Technology. Charlotteswille, 1996. P. 349-355.

76. Naftaly M. and Miles R.E. Terahertz time-domain spectroscopy for material characterization. Proc. IEEE. 2007. 95, No. 8. P. 1658-1665.

77. Grichkowsky D., Keiding S., Van Exter M., and Fattinger Ch. Far-infrared time-domain spectroscopy with terahertz beams of dielectric and semiconductors. J. Opt. Soc. Amer. 1990. 7, No. 10. P. 2006-2015.

78. Chen J., Chen Yu., Zhao H., Bastiaans G.J., and Zhang X.-C. Absorption coefficients of selected explosives and related compounds in the range of 0.1-2.8 THz. Opt. Express. 2007. 15, No. 19. P. 12060-12067.

79. Siebert K., Loffler T., Quast H., Thomson M., Bauer T., Leonhardt R., Czasch S. and Roskos H.G. All-optoelectronic continuous wave $\mathrm{THz}$ imaging for biomedical applications. Phys. Med. Biol. 2002. 47. P. 3743-3748. 